\title{
LARP7 is a potential tumor suppressor gene in gastric cancer
}

\author{
Yulan Cheng ${ }^{1}$, Zhe Jin ${ }^{1}$, Rachana Agarwal ${ }^{1}, \mathrm{Ke} \mathrm{Ma}^{2}$, Jian Yang ${ }^{1}$, Soibrahim Ibrahim ${ }^{1}$, Alexandru V Olaru', \\ Stefan David ${ }^{1}$, Hassan Ashktorab ${ }^{3}$, Duane T Smoot ${ }^{3}$, Mark D Duncan ${ }^{4}$, David F Hutcheon', John M Abraham¹, \\ Stephen J Meltzer ${ }^{1, \star}$ and Yuriko Mori ${ }^{1, *}$
}

We previously reported frequent truncating mutations of the RNA-binding protein gene, La ribonucleoprotein domain family, member-7 (LARP7) in gastric cancers (GCs) with frequent microsatellite instability. LARP7 negatively regulates positive transcription elongation factor-b ( $p-T E F b$ ) by binding to and stabilizing 7sk RNA. $p-T E F b$ has been linked to proliferation and de-differentiation in various tissues. Therefore, we reasoned that loss of LARP7 may contribute to gastric tumorigenesis. In this study, we evaluated LARP7 mRNA expression in $18 \mathrm{GCS}$, their corresponding non-neoplastic gastric tissues $\left(\mathrm{N}_{\mathrm{GC}}\right)$, and 18 normal gastric tissues from healthy individuals $\left(\mathrm{N}_{\mathrm{N}}\right)$. We also assessed the effects of transient small interfering (siRNA)-mediated LARP7 knockdown in immortalized non-neoplastic gastric epithelial cells. LARP7 mRNA was significantly decreased in GCs (median 2.5) relative to $N_{N} S$ (median 14.9, $P<0.01$ ) as well as relative to their corresponding $\mathrm{N}_{\mathrm{GC}} \mathrm{S}$ (median 8.1, $P<0.01$ ). Transfection of an siRNA directed against LARP7 (anti-LARP7 siRNA) into nonneoplastic gastric epithelial cells decreased 7 sk levels by $72 \%$ relative to a control siRNA $(P<0.01)$. Furthermore, antiLARP7 siRNA transfection increased cell proliferation by $23 \%(P<0.01)$ and cell migration by $22 \%(P<0.001)$ relative to control siRNA transfection. Taken together, these findings suggest that LARP7 downregulation occurs early during gastric tumorigenesis and may promote gastric tumorigenesis via p-TEFb dysregulation.

Laboratory Investigation (2012) 92, 1013-1019; doi:10.1038/labinvest.2012.59; published online 9 April 2012

KEYWORDS: gastric cancer; LARP7; p-TEFb; RNA-binding protein; 7sk snRNA

Gastric adenocarcinoma (GC) is a major cause of cancer-related death worldwide, ranking second only to lung cancer. ${ }^{1}$ There are approximately 750000 new cases diagnosed annually around the world, and 5-year overall survival rates are $<25 \%$. ${ }^{1}$ It is therefore important to increase our understanding of the molecular events underlying GC, not only to improve early detection, but also to aid in the development of more effective interventions. Unfortunately, precise mechanisms giving rise to the malignant transformation of gastric epithelial cells are not yet fully understood, awaiting the revelation of additional genes and pathways unique to this deadly disease. ${ }^{2}$

We previously reported frequent frameshift mutation of a novel gene, La ribonucleoprotein domain family, member-7 (LARP7, also referred to as HDCMA18P or PIP7S), in a subset of human GC. ${ }^{3}$ LARP7 belongs to the LARP RNA-binding protein family, which includes the lupus LA antigen gene, and modulates the metabolism and function of a variety of RNA species. ${ }^{4}$ LARP7 binds to and stabilizes 7sk snRNA. ${ }^{5,6}$ In turn, the 7sk-LARP7 complex suppresses the positive transcription elongation factor $\mathrm{b}(\mathrm{p}-\mathrm{TEFb})$ complex. The $\mathrm{p}-\mathrm{TEFb}$ complex, consisting of CDK9 and cyclin T1, is the ubiquitous and principal promoter of general mRNA elongation and processing. ${ }^{5,7} \mathrm{p}$-TEFb is a crucial mediator of c-Myc-mediated transcriptional activation, ${ }^{8}$ and $\mathrm{p}$-TEFb hyperactivity promotes malignant transformation of fibroblasts in vitro. ${ }^{9}$ Furthermore, LARP7 deletion has been reported to result in transformation of a cultured mamillary epithelial cell line via p-TEFb activation. ${ }^{5}$ Thus, these are possible pathways and

\footnotetext{
${ }^{1}$ Division of Gastroenterology, Department of Medicine, Johns Hopkins University School of Medicine, Baltimore, MD, USA; ${ }^{2}$ University Maryland at College Park, Baltimore, MD, USA; ${ }^{3}$ Division of Gastroenterology, Department of Medicine and Cancer Center, Howard University College of Medicine, Washington , DC, USA and ${ }^{4}$ Department of Surgery, Johns Hopkins Bayview Medical Center, Baltimore, MD, USA

Correspondence: Dr Y Mori, MD, PhD, Department of Medicine, Johns Hopkins University School of Medicine, 720 Rutland Avenue, Ross 945, Baltimore, MD 21205, USA or Professor SJ Meltzer, MD, 1503 E Jefferson Street Room\#112, Baltimore, MD 21231, USA.

E-mail: ymori3@jhmi.edu or smeltzer@jhmi.edu

*These two authors are co-senior authors.
} 
mechanisms through which LARP7 may exert tumor-suppressive effects in the stomach.

In order to assess potential tumor-suppressive properties of the LARP7 gene in the stomach, we first evaluated LARP7 expression in non-neoplastic and neoplastic gastric tissues. We then investigated functional effects of small interfering RNA (siRNA)-mediated LARP7 inhibition in immortalized non-neoplastic gastric epithelial cells.

\section{MATERIALS AND METHODS Materials}

HFE145, an immortalized human non-neoplastic gastric epithelial cell line, was previously established by $\mathrm{H}$ Ashktorab and DT Smoot. ${ }^{10,11}$ In this study, 18 primary GCs and their corresponding non-neoplastic gastric mucosal tissues (referred to as $\mathrm{N}_{\mathrm{GC}} \mathrm{s}$ ) were obtained at surgery from GC patients who had not undergone any neoadjvant therapy. Surgically resected tissues were macroscopically dissected to eliminate serosal and muscular layers before cryostorage. An additional 18 normal gastric mucosal tissues were obtained from individuals who underwent endoscopy for asymptomatic Barrett's esophagus surveillance and exhibited no abnormalities in the stomach. Written informed consent was obtained from all the participants according to a protocol approved by the Johns Hopkins University School of Medicine Institutional Review Board. Clinical and pathological characteristics are summarized in Table 1.

\section{Real-Time Quantitative RT-PCR (qRT-PCR)}

Total RNA was extracted using Trizol reagent (Invitrogen, Carlsbad, CA, USA) and treated with RNase-free DNase. First-strand cDNA was synthesized from $1 \mu \mathrm{g}$ of total RNA using a RevertAid cDNA synthesis kit (Fermentas, Glen Burnie, MD, USA). qRT-PCR was performed using iQ SYBR Green Supermix or iQ Probe Supermix on an iQ5 Multicolor Real-Time PCR Detection System (Bio-Rad, Hercules, CA, USA). $\beta$-Actin and GAPDH were used as normalization controls for LARP7 mRNA and 7sk snRNA, respectively. Methods of calculating RNA expression levels using these controls were described previously. ${ }^{12}$ Duplicate experiments were performed for each LARP7 mRNA measurement. For $7 \mathrm{sk}$ snRNA measurement, two independent experiments were performed, each of which was carried out in triplicate. The sequences of all primers and probes are shown in the Table 2 .

\section{Immunohistochemical Staining}

In all, 5 - $\mu \mathrm{m}$-thick sections of formaldehyde-fixed and paraffinembedded gastric tissues were used for immunohistochemical staining. Antigen retrieval was performed in citrate buffer $(\mathrm{pH}$ 6.0) according to the standard protocol. Immunohistochemical staining was carried out using rabbit polyclonal anti-human LARP7 antibody (1:400 dilution; LARP7-101AP, Fab Gennix, Frisco, TX, USA) or negative control antibody (normal rabbit IgG, Cell Signaling, Danvers, MA, USA) as the primary antibody. Visualization of protein was carried out using an
Table 1 Patient clinical and demographical data

\begin{tabular}{|c|c|c|c|}
\hline Case class & Gastric cancer & Control & $P^{a}$ \\
\hline Number & 18 & 18 & \\
\hline Age: mean (s.d.) & $66.3(16.2)$ & $56.2(19.6)$ & 0.10 \\
\hline Gender & & & 1.00 \\
\hline $\mathrm{F}$ & 8 & 9 & \\
\hline M & 10 & 9 & \\
\hline Race & & & 0.38 \\
\hline Black & 4 & 2 & \\
\hline White & 11 & 15 & \\
\hline Other/unavailable & 3 & 1 & \\
\hline Tumor stage & & & NA \\
\hline I & 2 & NA & \\
\hline$\|$ & 4 & NA & \\
\hline III & 4 & NA & \\
\hline IV & 6 & NA & \\
\hline Unavailable & 2 & NA & \\
\hline Tumor histology & & & NA \\
\hline Intestinal & 11 & NA & \\
\hline Diffuse & 6 & NA & \\
\hline Unavailable & 1 & NA & \\
\hline
\end{tabular}

Abbreviation: NA, not applicable.

${ }^{\text {a }}$ Student's $t$-test $P$-value for age, and Fisher's exact test $P$-values for gender and race comparison.

UltraVision Detection System Anti-Rabbit HRP kit (Lab Vision, Kalamazoo, MI, USA) and an anti-rabbit IgG secondary antibody (PowerVision Poly HRP anti-rabbit IgG; Leica Microsystems, Buffalo Grove, IL, USA), according to the manufacturer's protocol. Slides were then counterstained with hematoxylin and examined with an Olympus BX41 photomicroscope (Olympus, Center Valley, PA, USA). All immunohistochemically analyzed cases were included in the cohort used for qRT-PCR analysis.

\section{siRNA Transfection}

An siRNA duplex directed against LARP7 (anti-LARP7 siRNA) as well as a non-targeting control siRNA (control siRNA, D-001210-05; both from Dharmacon, Lafayette, CO, USA) were used. HFE- 145 cells were seeded onto six-well plates at a density of $2 \times 10^{5}$ cells per well. After $24 \mathrm{~h}$ of culture, siRNA was transfected at a final concentration of $60 \mathrm{nM}$ using Lipofectamine RNAiMax (Invitrogen), according to the manufacturer's protocol. LARP7 and 7sk snRNA expression levels were measured at $48 \mathrm{~h}$ after transfection. Three independent 
Table 2 Primer, probe, and siRNA sequences

\begin{tabular}{llll}
\hline Gene & Forward primer sequence $\left(5^{\prime}-3^{\prime}\right)$ & Reverse primer sequence $\left(5^{\prime}-3^{\prime}\right)$ & TagMan probe sequence $\left(5^{\prime}-3^{\prime}\right)$ \\
\hline LARP7 & TITGCGTTGTGGAATTGA & AGGCTGGAATGGGCTTATT & \\
7 sk & GACATCTGTCACCCCATTGA & GCGCAGCTACTCGTATACCC & CGATAGAGGAGGACCGGTCT \\
GAPDH & CAGCCTCAAGATCATCAGCA & TGTGGTCATGAGTCCTTCCA & \\
$\beta$-Actin & ACCATGGATGATGATATCGCC & GCCTTGCACATGCCGG & CGCTCGTCGTCGACAACGACGGC
\end{tabular}

LARP7 siRNA target sequence

Sense sequence

Antisense sequence
NNGAAGAAAGGCCGAAUGAAA

NNGAAGAAAGGCCGAAUGAAA

UUUCAUUCGGCCUUUCUUCUU
siRNA transfections and triplicate RNA measurements were carried out for each experimental condition.

\section{Western Blotting}

Cells were lysed in $100 \mu \mathrm{l}$ of cell lysis buffer ( $\mathrm{NaCl}: 149 \mathrm{mM}$, Nonidet P-40: $0.01 \%$, Tris: $50 \mathrm{mM}, \mathrm{pH} 7.8$, and protease inhibitor cocktail: $0.5 \%$ (Sigma, St Louis, MO, USA). Protein concentration was determined using a BCA Protein Assay Kit (Pierce, Rockford, IL, USA) with human serum albumin as a standard. In all, $30 \mu \mathrm{g}$ of each sample was loaded into each well of a 10\% TRIS-HCL gel (Bio-Rad). After electrophoresis, protein was transferred onto a PVDF membrane (Millipore, Bedford, MA, USA). The membranes were immunoblotted with TBS containing $5 \%$ nonfat dry milk, washed with $0.1 \%$ TBST and probed with 1:2000 anti-human LARP7 rabbit polyclonal antibody (LARP7-101AP, Fab Gennix) and 1:10000 anti-human $\beta$-actin mouse monoclonal antibody (Cat\# A3854, Sigma-Aldrich, Bedford, MA, USA), respectively. Horseradish peroxidase-conjugated anti-rabbit goat IgG (1:5000) (Calbiochem, Cat\# 401393, San Diego, CA, USA) and anti-mouse IgG (Invitrogen, Cat\# 626520, Camarillo, CA, USA) and an ECL Western Blotting detection kit (Amersham Pharmacia Biotech, Piscataway, NJ, USA) were used for target protein visualization. LARP7 protein levels were measured by densitometory and normalized to $\beta$-actin protein levels.

\section{Cell Proliferation Assay}

HFE-145 cells were re-seeded onto 96-well plates at $24 \mathrm{~h}$ after siRNA transfection at a density of 1000 cells per well (day 0 ). Cell proliferation was assessed at day 0 , day 2 and day 5, using the cell proliferation reagent WST-1 (Roche, Mannheim, Germany). In all, $10 \mu \mathrm{l}$ of reagent was added to each well, incubated at $37^{\circ} \mathrm{C}$ for $1 \mathrm{~h}$, and optical density was measured at $660 \mathrm{~nm}$ (background) and $440 \mathrm{~nm}$ (signal) using a plate reader (Molecular Devices, Sunnyvale, CA, USA). Independent experiments were repeated three times, with six replicates in each experiment. Three independent siRNA transfections and triplicate measurements were carried out for each experimental condition.

\section{Cell Migration and Invasion Assays}

Cell migration was measured by using transwell chamber plates (24-well format, BD Biosciences, St Louis, MO, USA), as described previously. ${ }^{13}$ Briefly, $5 \times 10^{4}$ cells were seeded onto a Transwell insert at $48 \mathrm{~h}$ after siRNA transfection. Twenty percent fetal bovine serum was used as a chemoattractant. After 22 -h incubation at $37^{\circ} \mathrm{C}$, cells that did not migrate through the pores of the transwell inserts were manually removed with a cotton swab. Cells present at the bottom of the membrane were fixed in cold methanol for $10 \mathrm{~min}$ and then stained with $0.01 \%$ crystal violet in $20 \%$ ethanol. After $10 \mathrm{~min}$ of incubation, the filters were washed thoroughly in water and suspended in $300 \mu \mathrm{l}$ of $5 \%$ acetic acid and 5\% methanol. Colorimetric readings were taken at OD $595 \mathrm{~nm}$. Independent experiments were repeated three times, with triplicates in each experiment. Invasion assays were performed in a similar fashion as migration assays, except that cells were seeded onto Matrigel-coated transwell chamber plates. ${ }^{14}$ Two independent experiments were performed, with triplicates in each experiment.

\section{Statistical Analysis}

Mean and standard error values are shown as representative values for data in figures, unless otherwise stated. Quantitative data for transfection experiments were normalized to the mean value of mock transfectants, unless otherwise stated. Student's $t$-test or one-way ANOVA testing were used for statistical evaluation of continuous variables, unless otherwise noted. Fisher's exact test or $\chi^{2}$ testing was used for statistical evaluation of categorical data. LARP7 mRNA expression in tissues did not follow a normal distribution, thus statistical assessment in tissues was achieved using the nonparametric Mann-Whitney $U$-test, Kruskal-Wallis test or 

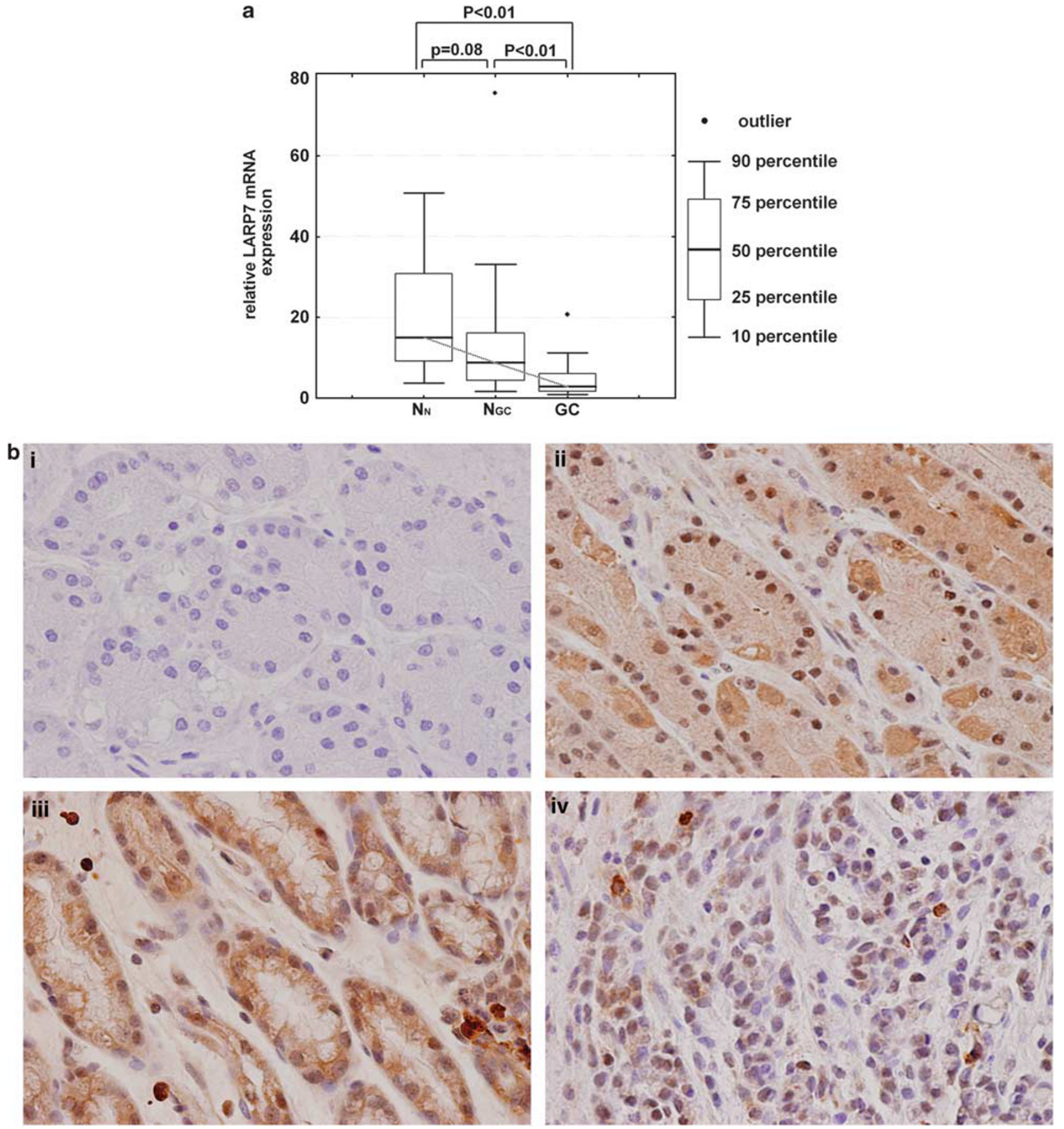

Figure 1 LARP7 is downregulated in primary gastric cancers. (a) The LARP7 mRNA levels were assessed in 18 normal gastric mucosae from healthy individuals $\left(\mathrm{N}_{N}\right)$ as well as 18 paired gastric cancer tumor tissues $(\mathrm{GC})$ and non-neoplastic gastric mucosae ( $\mathrm{N}_{\mathrm{GC}}$ ) by qRT-PCR. There were significant differences in LARP7 mRNA expression levels between these three tissue groups $(P<0.01$, Kruskal-Wallis test). LARP7 mRNA levels relative to the average of the $\mathrm{N}_{\mathrm{N}}$ specimens are shown in this graph. LARP7 mRNA was significantly reduced in GCs relative to $\mathrm{N}_{\mathrm{GC}} \mathrm{s}$ as well as to $\mathrm{N}_{\mathrm{N}} \mathrm{s}(P<0.01$ and $P<0.01$, respectively). There was an insignificant trend toward LARP7 mRNA downregulation in $\mathrm{N}_{\mathrm{GC}} \mathrm{S}$ relative to $\mathrm{N}_{\mathrm{N}} \mathrm{S}(P=0.08)$. (b) Immunohistochemical staining of LARP7 in representative gastric mucosal specimens $(400 \times)$. Panel i: Normal mucosa from a noncancer patient $\left(\mathrm{N}_{\mathrm{N}}\right)$ stained with negative control antibody. This slide shows no staining, assuring the specificity of the anti-LARP7 antibody (panels ii-iv). Panel ii: The same normal mucosal specimen shown in panel $\mathrm{i}$, stained with anti-LARP7 antibody. This slide displays abundant nuclear and cytoplasmic LARP7 protein expression that is restricted to gastric epithelial cells. Panel iii: Non-cancerous gastric mucosa from a cancer patient $\left(\mathrm{N}_{\mathrm{GC}}\right)$ stained with anti-LARP7 antibody. This slide shows abundant LARP7 staining of normal gastric epithelial cells. As in panel ii, very little LARP7 staining is observed in stromal cells. Panel iv: Infiltrating poorly differentiated adenocarcinoma from the same patient as panel iii stained with LARP7 antibody. This slide shows markedly reduced LARP7 staining in cancer relative to matching non-cancerous gastric mucosa from the same patient. 
Spearman's rank correlation. $P$-values $<0.05$ were used as a statistical significance cutoff.

\section{RESULTS}

LARP7 mRNA Is Significantly Downregulated in Cancerous Gastric Mucosa Relative to Non-Neoplastic Gastric Mucosae

We first investigated the potential association of LARP7 mRNA levels with neoplastic status of human gastric mucosal tissues. For this purpose, we analyzed 18 gastric cancers (GCs), their corresponding non-neoplastic gastric mucosal tissues $\left(\mathrm{N}_{\mathrm{GC}}\right)$ and 18 normal gastric mucosal tissues from healthy individuals $\left(\mathrm{N}_{\mathrm{N}}\right)$. Cancer patients tended to be older than healthy individuals, but this tendency did not reach statistical significance $(66.3 \pm 16.2$ vs $56.2 \pm 19.6$ years of age, $P=0.1$; Table 1). No significant difference in gender or race was observed between cancer cases and healthy individuals (Table 1).

LARP7 mRNA expression was significantly reduced in GCs (median 2.5) relative to $\mathrm{N}_{\mathrm{GC}} \mathrm{s}$ (median $8.1, P<0.01$ ) or $\mathrm{N}_{\mathrm{N}} \mathrm{S}$ (median 14.9, $P<0.01$; Figure 1a). LARP7 mRNA expression also showed a trend toward reduction in $\mathrm{N}_{\mathrm{GC}} \mathrm{S}$ 8.1) vs $\mathrm{N}_{\mathrm{N}} \mathrm{s}$ (14.9, $P=0.08$; Figure 1a). LARP7 mRNA expression in GCs was not significantly associated with histological subtype (ie, intestinal $v s$ diffuse type) or distant metastasis. LARP7 mRNA expression was significantly and inversely correlated with age in $\mathrm{N}_{\mathrm{GC}}$ s (Spearman rank correlation coefficient $-0.51, P=0.02$ ) but not in $\mathrm{N}_{\mathrm{N}} \mathrm{s}$ (Spearman rank correlation coefficient $-0.36, P=0.14$ ) or GCs (Spearman rank correlation coefficient $-0.30, P=0.22$ ). As there was a nonsignificant but large difference in age between $\mathrm{N}_{\mathrm{GC}} \mathrm{s}$ and $\mathrm{N}_{\mathrm{N}} \mathrm{s}$, we further assessed only cases older than 50 years of age. In this age-matched comparison, the nonsignificant trend toward decreased LARP7 mRNA expression in $\mathrm{N}_{\mathrm{GC}} \mathrm{S}$ was not detected $(P=0.42)$. LARP7 mRNA expression was not significantly correlated with gender or race in any of the three tissue categories (data not shown).

\section{Immunohistochemical Staining Confirms LARP7 Protein Expression in Gastric Epithelial Cells}

We further examined LARP7 protein expression patterns in the stomach by immunohistochemical staining (Figure 1b). Normal gastric mucosae from noncancer patients (ie, $\mathrm{N}_{\mathrm{N}}$ ) showed abundant nuclear and cytoplasmic LARP7 protein staining in epithelial cells, while stromal cells rarely demonstrated any LARP7 staining. Reduced LARP7 protein levels were observed in adenocarcinomas (ie, GCs) relative to their corresponding non-cancerous gastric mucosae (ie, $\mathrm{N}_{\mathrm{GC}} \mathrm{S}$ ), consistent with results shown in Figure 1a. As in $\mathrm{N}_{\mathrm{N}} \mathrm{s}$, stromal cells in $\mathrm{N}_{\mathrm{GC}} \mathrm{s}$ did not demonstrate marked LARP7 staining.

\section{LARP7 Knockdown Decreases 7sk snRNA Quantity in Gastric Epithelial Cells}

The putative tumor-suppressive function of LARP7 is repression of the tumor-promoting transcriptional elongation a
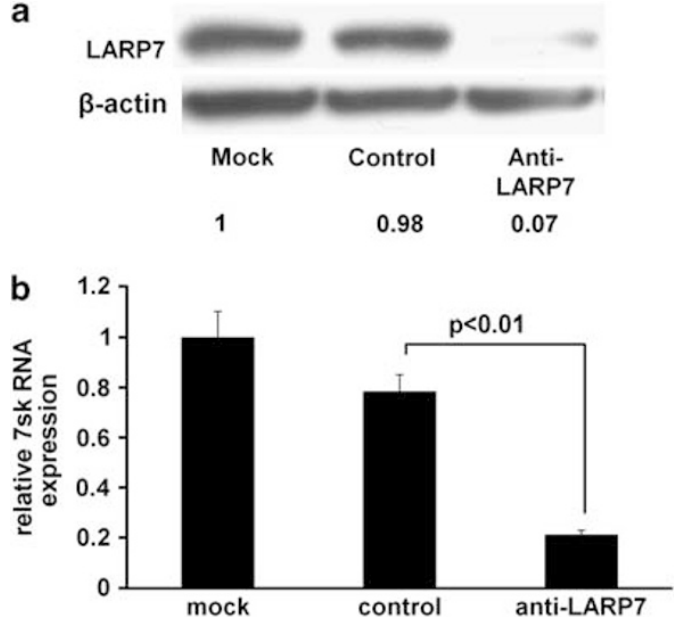

Figure 2 LARP7 knockdown decreases the 7sk snRNA quantity in nonneoplastic gastric epithelial cells. (a) LARP7 protein levels were assessed by western blot in HFE145 cells. A marked decrease in LARP7 is evident in antiLARP7 siRNA transfectants relative to mock and control siRNA transfectants. (b) 7sK snRNA levels were measured for mock-, anti-LARP7 siRNA-, and control siRNA-transfected HFE145 cells. The 7sk snRNA data relative to the average of the mock transfectant data are shown in this graph. A significant reduction of 7sK level was observed in anti-LARP7 siRNA transfectants relative to control siRNA- and mock-transfected cells $(P<0.01$ and $P<0.01$, respectively).

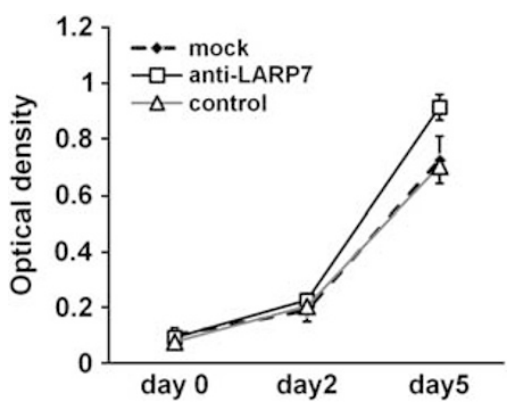

Figure 3 LARP7 knockdown increases non-neoplastic gastric epithelial cell proliferation in vitro. Cell proliferation levels were measured by WST-1 assay. There was a significant difference in cell proliferation between mock-, non-specific control siRNA-, and anti-LARP siRNA-transfected cells $(P<0.01$, one-way ANOVA test). Anti-LARP7 siRNA significantly increased growth rates in HFE145 cells, compared to no specific control siRNA and mock conditions $(P<0.01$ and $P<0.01$, respectively).

complex, p-TEFb: LARP7 binds to and stabilizes 7sk snRNA, thus constituting the principal negative regulator of $\mathrm{p}-\mathrm{TEFb}$, $7 \mathrm{sk}$ snRNP. ${ }^{15}$ In order to verify the involvement of LARP7 in the negative regulation of $\mathrm{p}$-TEFb via $7 \mathrm{sk}$ snRNA stabilization in the gastric epithelium, we analyzed the impact of siRNA-mediated LARP7 knockdown on 7sk snRNA quantity in an immortalized non-neoplastic gastric epithelial cell line, HFE145. LARP7 protein level was reduced by $95 \%$ in antiLARP7 siRNA vs control siRNA transfectants (Figure 2a). 7sk mRNA levels were significantly reduced in anti-LARP7 


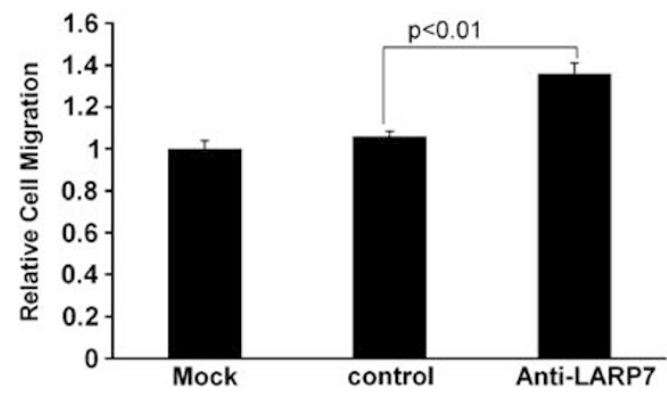

Figure 4 LARP7 knockdown promotes non-neoplastic gastric epithelial cell migration in vitro. Cell migration levels were assessed for mock-, anti-LARP7 siRNA-, and control siRNA-transfected HFE145 cells at $48 \mathrm{~h}$ after transfection using the transwell assay. There was a significant difference among these three groups of cells $(P<0.01$, one-way ANOVA test). Cell migration increased by 1.4-fold in anti-LARP7 siRNA transfectants relative to control transfectants $(P<0.01)$.

siRNA transfectants $(0.21 \pm 0.02)$ relative to control siRNA transfectants $(0.78 \pm 0.07, P<0.01$; Figure $2 b)$.

\section{LARP7 Knockdown Increases Gastric Epithelial Cell Proliferation In Vitro}

We then evaluated effects of LARP7 knockdown on HFE145 cell proliferation by using WST-1 assays. Cells were transfected with anti-LARP7 siRNA, control siRNA or mock reagents on day -1 , and the WST- 1 assay was performed on day 0 , day 2 and day 5 . On day 5, we observed a significant increase in cell proliferation in anti-LARP7 siRNA transfectants $(0.91 \pm 0.05)$ relative to control siRNA transfectants $(0.70 \pm 0.03, P<0.001$; Figure 3).

\section{LARP7 Knockdown Promotes Gastric Epithelial Cell Migration In Vitro}

Increased cell motility is one of the properties shared by cancer cells arising in multiple organs. Therefore, we also investigated whether LARP7 knockdown influences gastric epithelial cell motility. For this purpose, we conducted transwell assays of HFE145 cells at $48 \mathrm{~h}$ after transfection with anti-LARP7 siRNA, control siRNA, and mock reagent transfection. As shown in Figure 4, anti-LARP7 siRNA transfectants demonstrated significantly more migrating cells (1.36 \pm 0.21 ,) than did control siRNA transfectants (1.06 \pm 0.09 , $P<0.001)$. We also assessed the impact of LARP7 knockdown on HFE 145 cell invasion. However, there was no significant difference in invasion between anti-LARP7 siRNA transfectants $(0.089 \pm 0.01$,$) and control siRNA transfectants$ $(0.088 \pm 0.01, P=0.78)$.

\section{DISCUSSION}

We previously discovered frequent truncating mutations of the LARP7 gene in microsatellite-unstable (MSI-H) GCs. ${ }^{3}$ In order to further evaluate the potential tumor-suppressive properties of this gene in GC, we measured LARP7 expression levels in gastric mucosae of normal and cancerous tissues, as well as the cell biological effects of LARP7 knockdown in vitro. Our data, to our knowledge, are the first to reveal that LARP7 mRNA expression is significantly reduced in human cancer relative to normal tissues, supporting our hypothesis that LARP7 is a tumor-suppressor gene. In our immunohistochemical analyses, LARP7 protein staining was overwhelmingly localized to gastric epithelial cells, rather than to stromal cells (including inflammatory infiltrates). Therefore, LARP7 downregulation in GCs relative to corresponding noncancerous gastric mucosae observed in snap-frozen tissues likely reflects LARP7 downregulation in cancerous gastric epithelial cells. We observed nuclear and cytoplasmic LARP7 protein expression in normal gastric epithelial cells, consistent with LARP7s known function and reported immunohistochemical staining pattern (Human Protein Atlas). ${ }^{16}$ We also performed transient LARP7 knockdown in a non-neoplastic gastric epithelial cell line, which resulted in a significant increase in cell proliferation and migration. Taken together, these data suggest that LARP7 downregulation contributes to the genesis of human GC.

LARP7 constitutes the specific and principal binding partner of 7 sk snRNA, capturing $>90 \%$ of $7 \mathrm{sk}$ snRNA. ${ }^{17}$ In this study, we verified that loss of LARP7 results in significant and substantial loss of 7sk snRNA, and by extrapolation, loss of $\mathrm{p}$-TEFb repression in gastric epithelial cells. LARP7 frameshift mutation, the prevalent mutant in MSI-H GCs reported by us, ${ }^{3}$ lacks the 21 C-terminal amino acids, whose loss abolishes LARP7s 7sk binding capacity. ${ }^{17,18}$ Therefore, MSI-H GCs also are likely to lose LARP7-mediated $\mathrm{p}$-TEFb repression and, consequently, to acquire a more malignant phenotype.

Notably, multiple agents that target $\mathrm{p}-\mathrm{TEFb}$ are currently being tested as anticancer drugs, with promising outcomes. Examples include a phase I clinical trial of Seliciclib in advanced solid tumors, ${ }^{19}$ a phase I clinical trial of Flavopiridol in advanced solid tumors including $\mathrm{GCs}^{20}{ }^{20}$ and a phase II clinical trial of Flavopiridol in leukemia. ${ }^{21}$ Thus, it appears that LARP7 may also become a potential therapeutic target in GC.

We observed a trend toward LARP7 downregulation in non-neoplastic gastric mucosae from GC patients $v s$ normal stomach from healthy individuals. However, this trend was undetectable in an age-matched comparison, suggesting that the observed LARP7 downregulation in non-cancerous gastric mucosae from GC patients may be an aging-associated event. Nevertheless, this aging-associated event may still constitute a promoting factor at early stages of carcinogenesis, since aging increases GC incidence (SEER17; National Cancer Institute, Surveillance, Epidemiology, and End Results Program; http://seer.cancer.gov/faststats/).

This study has several limitations. Its relatively small cohort size provides limited power, which may lead to type II error, especially in analyses of associations between LARP7 expression and tumor stage or histological subtype. Data on intestinal metaplasia or $H$. pylori status of GC patients were unavailable in the majority of cases, thus we were unable to correlate these critical phenotypes with LARP7 expression. 
Finally, cell biologic studies were carried out on a single cell line, HFE145, since to our knowledge, no other human nonneoplastic gastric epithelial cells were known to be reliable in transfection experiments.

In summary, our molecular and cell biological analyses of the LARP7 gene suggest that this gene possesses characteristics typical of a gastrointestinal tumor-suppressor gene. The precise molecular functions of LARP7 are not yet fully understood, but its protein domain structure suggests potential roles as an RNA-binding and -regulatory protein in the nucleus. Further studies are warranted to explore the precise antitumor mechanisms of this novel gene and its potential application to the clinical care of patients.

\section{ACKNOWLEDGEMENTS}

We thank all nurses and technicians at the Johns Hopkins Greenspring Station Endoscopy Suite, the Johns Hopkins Outpatient Center Endoscopy Suite and the Johns Hopkins Bayview Medical Center Operating Room for their assistance with sample acquisition. This work was funded by U01CA084986 (National Institute of Cancer; YM), Senior Research Award (Crohn's and Colitis Foundation of America; YM), Wendy Will Case Cancer Fund (Wendy Will Case Cancer Fund; YM), Research Grant (Prevent Cancer Foundation YM) 01CA133012 (National Cancer Institute; SJM) and R01CA146799 (National Cancer Institute; SJM).

\section{DISCLOSURE/CONFLICT OF INTEREST}

The authors declare no conflict of interest.

1. Jemal A, Siegel R, Ward E, et al. Cancer statistics, 2006. CA Cancer J Clin 2006;56:106-130.

2. Hamilton JP, Sato F, Jin Z, et al. Reprimo methylation is a potential biomarker of Barrett's-Associated esophageal neoplastic progression. Clin Cancer Res 2006;12:6637-6642.

3. Jacobs JJ, van Lohuizen M. Polycomb repression: from cellular memory to cellular proliferation and cancer. Biochim Biophys Acta 2002;1602: 151-161.

4. Bayfield MA, Yang R, Maraia RJ. Conserved and divergent features of the structure and function of La and La-related proteins (LARPs). Biochim Biophys Acta 2010;1799:365-378.

5. Barboric $M$, Lenasi $T$, Chen $H$, et al. 7SK snRNP/P-TEFb couples transcription elongation with alternative splicing and is essential for vertebrate development. Proc Natl Acad Sci USA 2009;106:7798-7803.
6. Swarbreck D, Wilks C, Lamesch $\mathrm{P}$, et al. The Arabidopsis Information Resource (TAIR): gene structure and function annotation. Nucleic Acids Res 2008:36(Database issue):D1009-D1014.

7. Bres V, Yoh SM, Jones KA. The multi-tasking P-TEFb complex. Curr Opin Cell Biol 2008;20:334-340.

8. Kanazawa S, Soucek L, Evan G, et al. c-Myc recruits P-TEFb for transcription, cellular proliferation and apoptosis. Oncogene 2003;22:5707-5711.

9. Moiola C, De Luca P, Gardner K, et al. Cyclin T1 overexpression induces malignant transformation and tumor growth. Cell Cycle 2010;9:3119-3126.

10. Smoot DT AC, Barnes $P$, Brown $M$, et al. Human gastric epithelial cell lines derived from primary cultures of normal gastric epithelial cells. Gastroenterology 2000;118:A540.

11. Akhtar $\mathrm{M}$, Cheng $\mathrm{Y}$, Magno RM, et al. Promoter methylation regulates Helicobacter pylori-stimulated cyclooxygenase-2 expression in gastric epithelial cells. Cancer Res 2001;61:2399-2403.

12. Mori $Y$, Cai $K$, Cheng $Y$, et al. A genome-wide search identifies epigenetic silencing of somatostatin, tachykinin-1, and 5 other genes in colon cancer. Gastroenterology 2006;131:797-808.

13. Adiseshaiah P, Lindner DJ, Kalvakolanu DV, et al. FRA-1 proto-oncogene induces lung epithelial cell invasion and anchorage-independent growth in vitro, but is insufficient to promote tumor growth in vivo. Cancer Res 2007;67:6204-6211.

14. Agarwal R, Mori $Y$, Cheng $Y$, et al. Silencing of claudin-11 is associated with increased invasiveness of gastric cancer cells. PLoS One 2009;4:e8002.

15. He N, Jahchan NS, Hong E, et al. A La-related protein modulates 7SK snRNP integrity to suppress P-TEFb-dependent transcriptional elongation and tumorigenesis. Mol Cell 2008;29:588-599.

16. Uhlen $M$, Bjorling $E$, Agaton $C$, et al. A human protein atlas for normal and cancer tissues based on antibody proteomics. Mol Cell Proteomics 2005;4:1920-1932.

17. Biewenga $P$, Buist MR, Moerland PD, et al. Gene expression in early stage cervical cancer. Gynecol Oncol 2008;108:520-526.

18. Markert A, Grimm M, Martinez J, et al. The La-related protein LARP7 is a component of the 7SK ribonucleoprotein and affects transcription of cellular and viral polymerase II genes. EMBO Rep 2008;9:569-575.

19. Le Tourneau C, Faivre S, Laurence V, et al. Phase I evaluation of seliciclib (R-roscovitine), a novel oral cyclin-dependent kinase inhibitor, in patients with advanced malignancies. Eur J Cancer 2010; 46:3243-3250.

20. Rathkopf D, Dickson MA, Feldman DR, et al. Phase I study of flavopiridol with oxaliplatin and fluorouracil/leucovorin in advanced solid tumors. Clin Cancer Res 2009;15:7405-7411.

21. Karp JE, Smith BD, Levis MJ, et al. Sequential flavopiridol, cytosine arabinoside, and mitoxantrone: a phase II trial in adults with poor-risk acute myelogenous leukemia. Clin Cancer Res 2007;13(15 Pt 1): 4467-4473. 